\title{
Comparison of Quality of Life, Satisfaction with Life, and Satisfaction with Or- thopedic Shoes among Veterans with Lower Extremities Length Discrepancy Receiving Services and Not-receiving Services after Two Years of Follow-up
}

\section{ART ICLE INF O}

\section{Article Type}

Descriptive Study

\section{Authors}

Heidari Zh. ${ }^{1} M S c$,

Karimi A. ${ }^{1} M D$,

Yavari A. ${ }^{1} B S C$

Masoumi M. ${ }^{1} M D$,

Vali Z. ${ }^{1} B S c$,

Faraji E. $P h D$

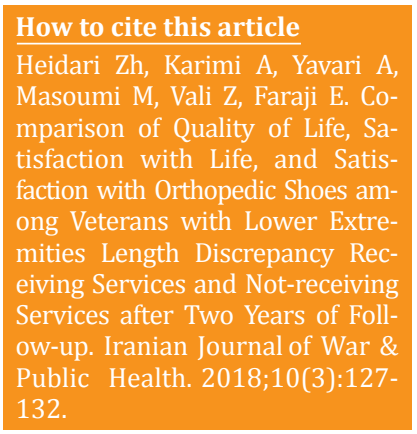

*Janbazan Medical and Engineering Research Center (JMERC), Tehran, Iran

${ }^{1}$ Janbazan Medical and Engineering Research Center (JMERC), Tehran, Iran

\section{Correspondence}

Address: Janbazan Medical \& Engineering Research Center (JMERC), No. 17, Farokh Street, Moghadas Ardabili Street, Yaman Street, Shahid Chamran Highway, Tehran, Iran. Postal Code: 1985946563

Phone: +98 (21) 22172424

Fax: +98 (21) 22418180

faraji.elahe.92@gmail.com

\section{Article History}

Received: July 03, 2017

Accepted: January 01, 2018

ePublished: August 29, 2018

\section{A B S T RA C T}

Aims That the services should be presented suitably tailored to the status of individuals is of high importance in health needs assessment. The aim of this study was to compare the quality of life, satisfaction with life, and satisfaction with orthopedic shoes among veterans with lower extremities length discrepancy receiving services and not-receiving services after two years of follow-up.

Instruments \& Methods This descriptive study was conducted among veterans with lower extremities difference in West Azarbaijan Province, Iran in 2014 and followed-up in 2016. A total of 76 veterans were selected by purposeful sampling, 48 of whom received prescriptive services (group 1) and 28 of whom received no services (group 2). The research tools were a Quality of Life Questionnaire, Satisfaction with Life Scale, and a researcher-made satisfaction with orthopedic shoes questionnaire. The data were analyzed by SPSS 20, using Chi-square, independent t-test, dependent t-test, Wilcoxon, and Mann-Whitney U test.

Findings Of veterans, $76.6 \%$ who had received orthopedic shoes were satisfied. In group 1, the difference of the evaluation of 2014 and 2016 was significant in mental health and physical health, but it was not significant in the overall score of quality of life, while in group 2, the overall quality of life score decreased significantly. The two groups did not have a significant difference in life satisfaction.

Conclusion Most veterans who use orthopedic shoes are highly satisfied. Veterans who received services after a two year follow-up have higher levels of physical health, mental health, and quality of life compared with the veterans who did not use the services. The two groups do not differ in their levels of satisfaction with life.

Keywords Orthotic Devices; Ankle; Quality of Life; Satisfaction; Veterans

\section{CIT A T I O N L I N KS}

[1] Passport for the orthopedic boards and FRCS examination [2] Functional scoliosis caused by leg length discrepancy [3] Management of leg length inequality [4] Limb-Length Inequality: Assessment and treatment options [5] Atlas of orthoses and assistive devices [6] Questionnaire for persons with a transfemoral amputation (Q-TFA): Initial validity and reliability of a new outcome measure [7] Study of stump problems of lower limb amputation in war casualties [8] A theory of metamotivation: The biological rooting of the value-life [9] Impact of adolescent mental disorders and physical illnesses on quality of life 17 years later [10] The short form health survey (SF-36): Translation and validation study of the Iranian version [11] The reliability and validity of the satisfaction with life scale [12] Satisfaction with military dental care by active duty soldiers [13] Dental satisfaction survey at an expeditionary dental clinic in support of operations enduring freedom and Iraqi freedom [14] Health status and satisfaction with health care: A longitudinal study among patients served by the veterans health administration [15] VA medical system earns high customer satisfaction ratings [16] Satisfaction of janbazan and azadegan referring health center of janbazan foundation of Kerman city to rcceive medical services in the year 2003-2004 [17] Service satisfaction among war related bilateral lower limb amputation [18] Health-related quality of life in patients served by the department of veterans affairs: Results from the veterans health study [19] Quality of life in blind war survivors [20] Sociodemographic and health factors explaining emotional wellbeing as a quality of life domain of older people in Madrid [21] Evaluation of quality of life in psychiatric veterans of Isfehan [22] Quality of life in chemical war victims with sever pulmonary damage [23] Quality of life in chemical veterans 15 years after exposure to mustard gas [24] Modernization and postmodernization: Cultural, economic, and political change in 43 societies [25] Domains of perfectionism: Prevalence and relationships with perfectionism, gender, age, and satisfaction with life [26] Coping strategies and life satisfaction: Chronically ill patients' perspectives 
فيزيوتراييست و روانشناسى مورد ارزيابى و معاينه قرار گرفتند.

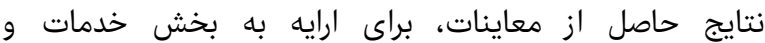

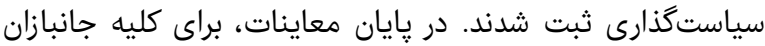

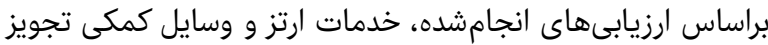

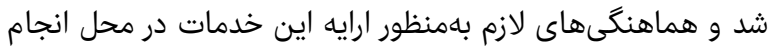

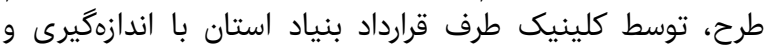

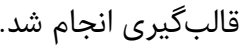

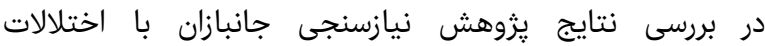

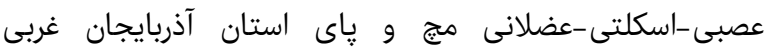

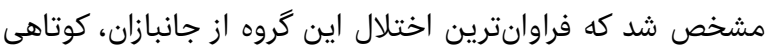

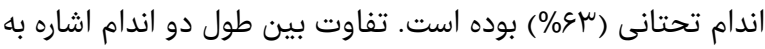

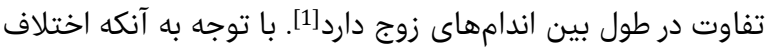

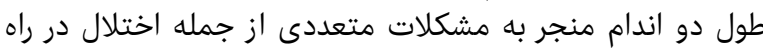

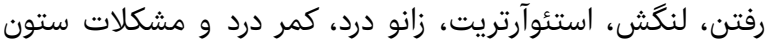

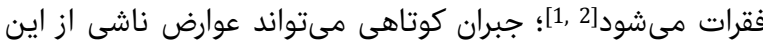

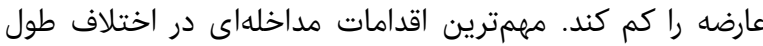

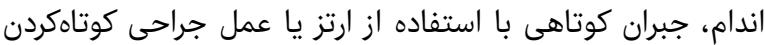

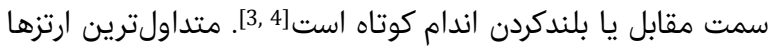

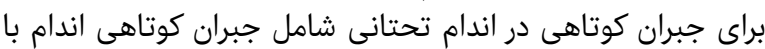

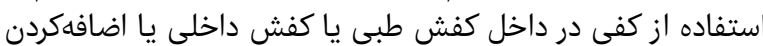

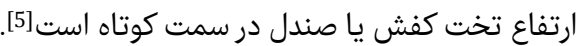

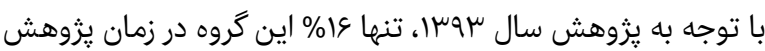

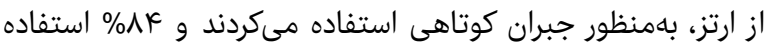

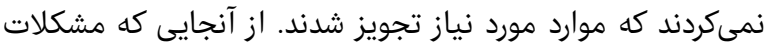

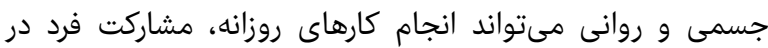

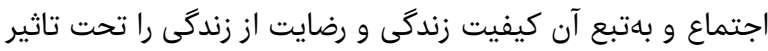

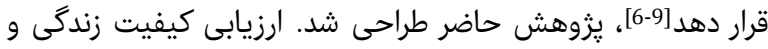

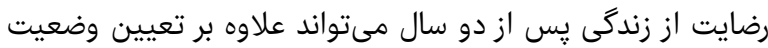

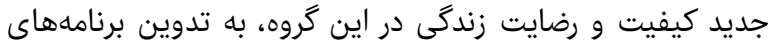

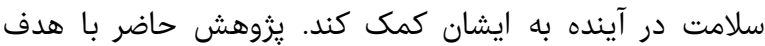

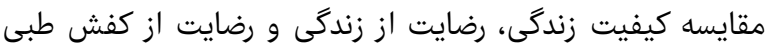

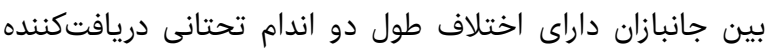

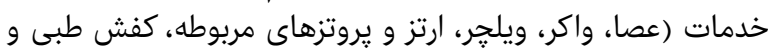

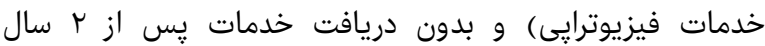

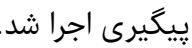

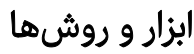

يزوهش مقطعى حاضر از نوع توصيفى-مقايساهاى بين جانبازازان

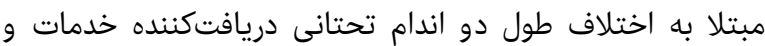

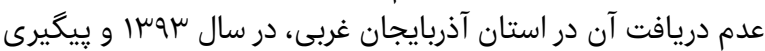

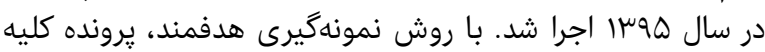

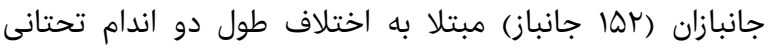

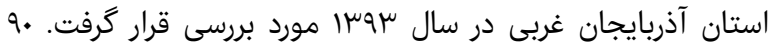

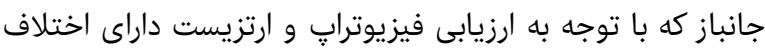

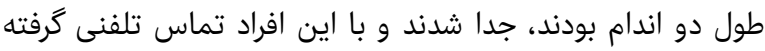

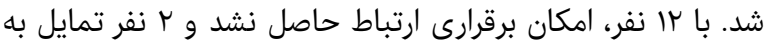

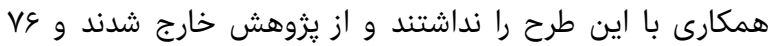

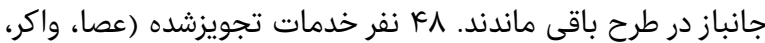

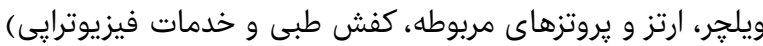

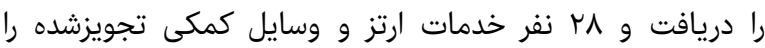
دريافت نكرده بودند. از فرمهاى بييگيرى مداخلات تجويزشده استفاده شد و اطلاعات

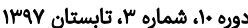

مقايسه كيفيت زندگى، رضايت از زندگى و رضايت

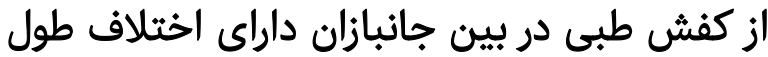

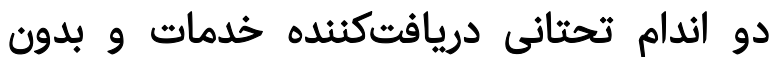
دريافت خدمات يس از دو سال بييكيرى دئي

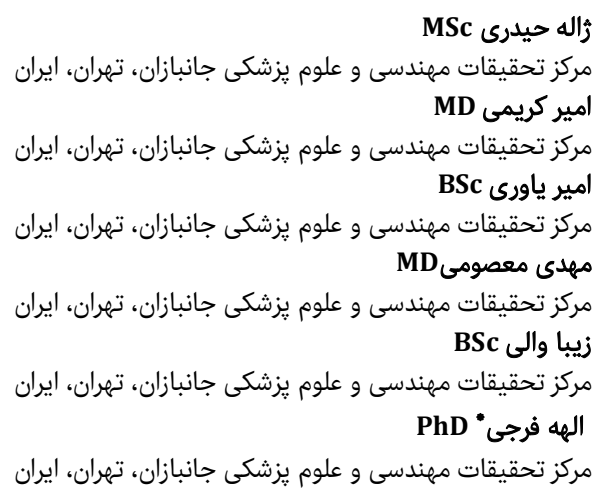

جكيده

اهداف: آنجه در نيازسنجى سلامت، مهم است اين است كه خدمات بهشكل

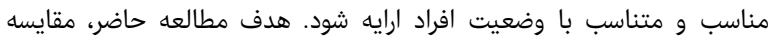

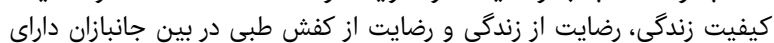

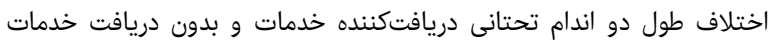

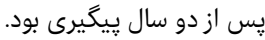

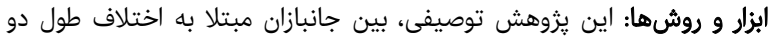

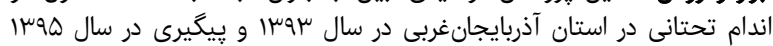

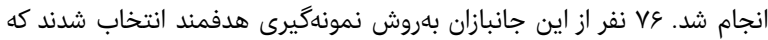

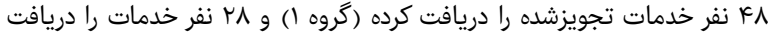

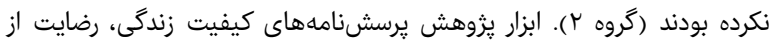

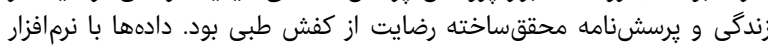
SPSS 20 من مويتنى يو تحليل شدند.

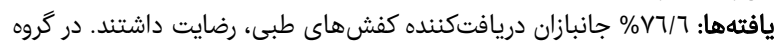

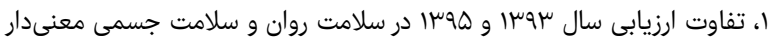

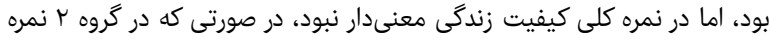

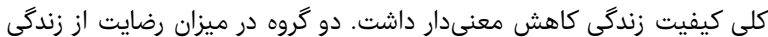

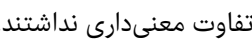

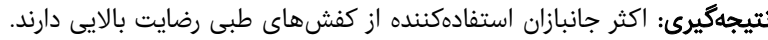

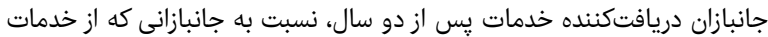

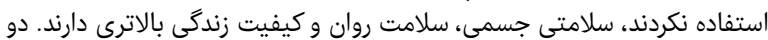

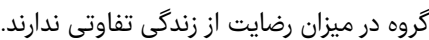

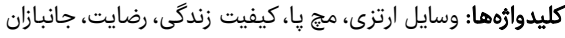

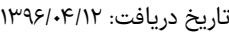

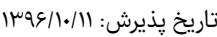

faraji.elahe.92@gmail.com : نويسنده مسئول بديرت:

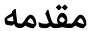

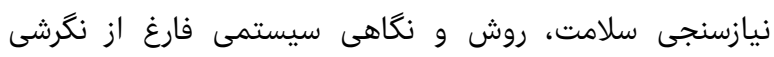

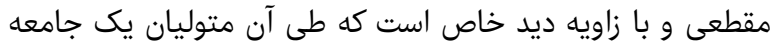

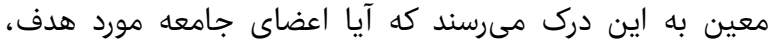

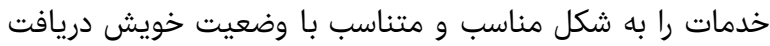

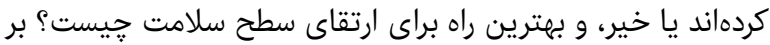

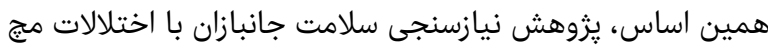

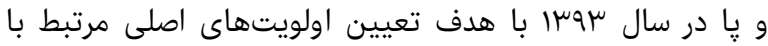

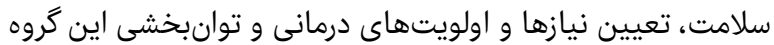

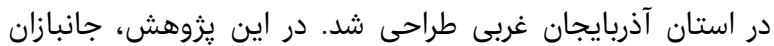

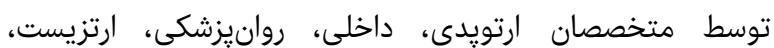

فصل نامه علمى - يزوهشى طب جانباز 
مقايسه كيفيت زندكى، رضايت از زندگى و رضايت از كفش طبى دربين جانبازان داراى اختلاف طول دو اندام تحتانى دريافتكننده ... وجا

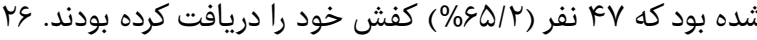
نفر (1/

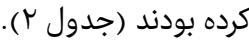

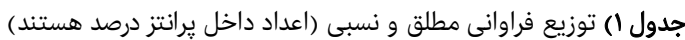

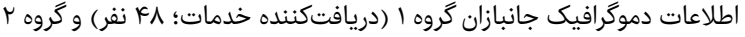
(بدون دريافت خدمات؛

\begin{tabular}{|c|c|c|}
\hline rروه r & كرو & متغيرها \\
\hline & & جنسيت \\
\hline rA $(1 . .1 \cdot)$ & $K \varphi(9 \Delta / \Lambda)$ & مرد \\
\hline - & $r(r / r)$ & زن \\
\hline & & وضعيت اشتغال \\
\hline$\varepsilon(r Y / K)$ & 19 (pq/9) & 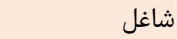 \\
\hline$r r(\vee \wedge / \varepsilon)$ & $r q(\varepsilon \cdot / k)$ & بدون شغل \\
\hline & & وضعيت تاهل \\
\hline - & $1(r / 1)$ & مجرد \\
\hline$r \wedge(1 . .1 \cdot)$ & $k V(9 V / 9)$ & متاهل \\
\hline & & سطح تحصيلات \\
\hline$\Lambda($ $\Lambda / \varepsilon)$ & $r V(\Delta \& / r)$ & زير ديبلم \\
\hline$\|(r q / \mu)$ & $18(\mu / \mu)$ & دييلم \\
\hline$q(\mu r / l)$ & $\Delta(1 \cdot / \Delta)$ & بالاتر از ديِيلم \\
\hline
\end{tabular}

جدول ץ) توزيع فراوانى مطلق و نسبى (اعداد داخل يرانتز درصد هستند) انواع

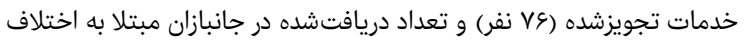
طول دو اندام تحتانى

\begin{tabular}{|c|c|c|}
\hline دريافتشده & تجويزشده & انواع خدمات \\
\hline$K V(\varnothing \Delta / r)$ & $V r(9 F / V)$ & كفش طبى معمولى با جبران كوتاهى \\
\hline$r \Delta(\Delta K / \mu)$ & $k 9(9 \cdot 10)$ & كفى طبى قالبگيرى با جبران كوتاهى \\
\hline is $(0 \cdot / \cdot)$ & $\mu r(K r / I)$ & زانوبند نئويرنى فنر دار \\
\hline$r(1 \cdot \bullet / \cdot)$ & $r(r / 8)$ & كفش ورزشى با كفى جبران كوتاهى \\
\hline$\Delta(\Lambda \mu / \mu)$ & $\varepsilon(V / 9)$ & بريس كوتاه \\
\hline $10(\Delta \mu / \varepsilon)$ & 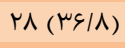 & كمربند طبى \\
\hline$F(0 \cdot 1 \cdot)$ & $\wedge(1 \cdot / \Delta)$ & عصاكراج \\
\hline$\mu(K r / A)$ & $V(q / r)$ & مجبند يا \\
\hline $1(1 . . / \cdot)$ & $1(1 / \mu)$ & يد ياشنه \\
\hline $1(0 \cdot / \cdot)$ & $r(r / 8)$ & كردنبند طبى \\
\hline $1(I r / \Delta)$ & $\Lambda(1 \cdot / 0)$ & ويلجر \\
\hline $1(11 / 1)$ & $9(11 / 1)$ & 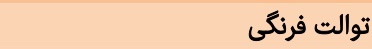 \\
\hline $1(1 \cdot .1 \cdot)$ & $1(1 / \mu)$ & سايورت آرنج \\
\hline - & $1(1 / \mu)$ & جوراب واريس \\
\hline • & $1(1 / \mu)$ & اسييلنت انكشت دست \\
\hline - & $1(1 / \mu)$ & اسيلنت اسيايدر \\
\hline - & $1(1 / \mu)$ & سايورت هيب \\
\hline
\end{tabular}

در گروه ا، تفاوت ارزيابى سال سوسا و سال هوسا در زيرمقياس

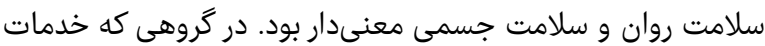

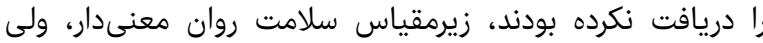

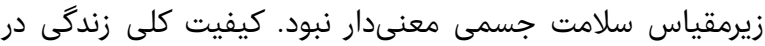

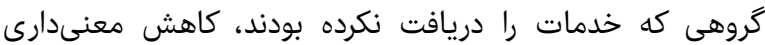

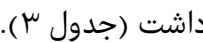

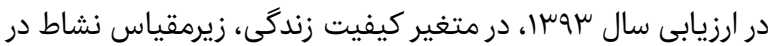

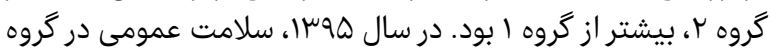

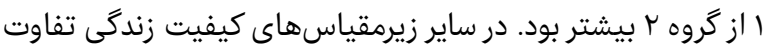

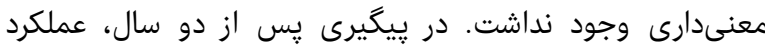

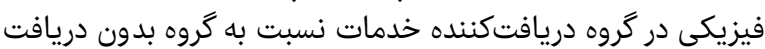

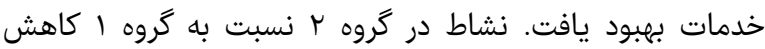
معنىدار بيشترى داشت (جدول ع).
جمعيتشناختى افراد شامل جنسيت، شغل، وضعيت تاهل،

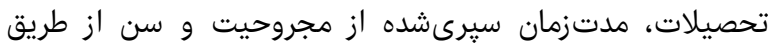

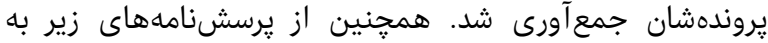

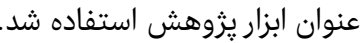

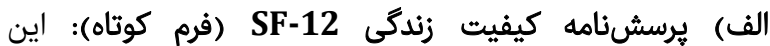

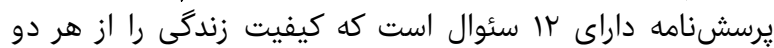

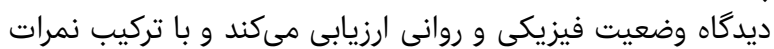

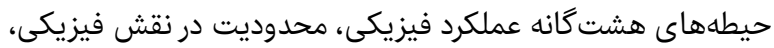

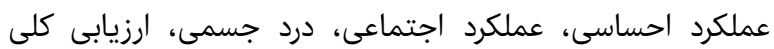

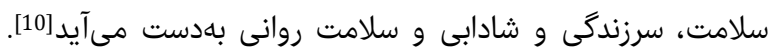

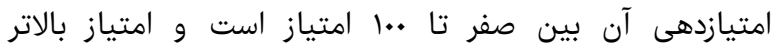

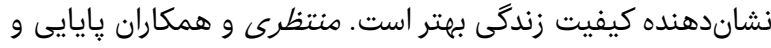

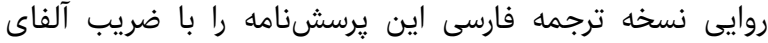

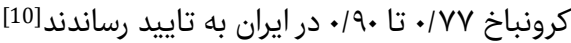

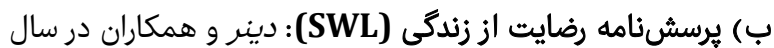

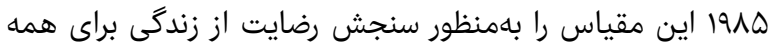

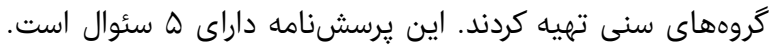

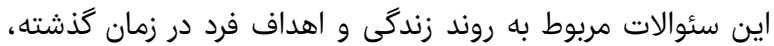

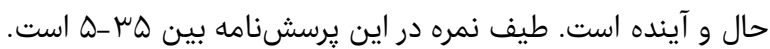

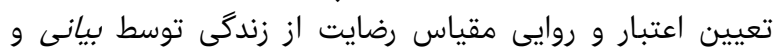

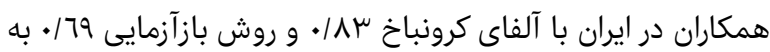

تاييد رسيده است[11]

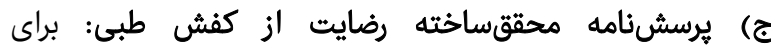

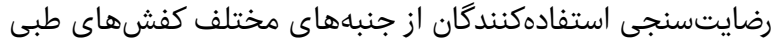

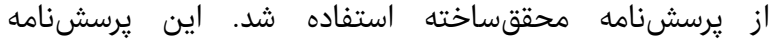

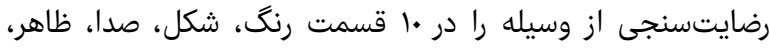

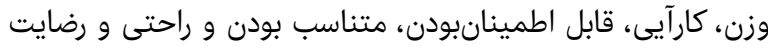

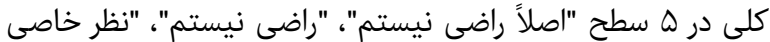

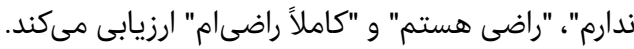

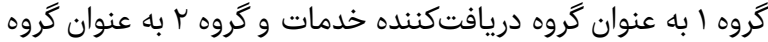

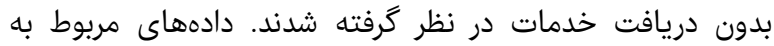

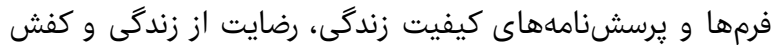

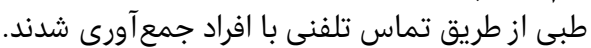

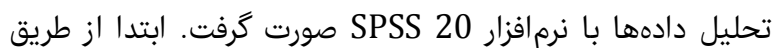

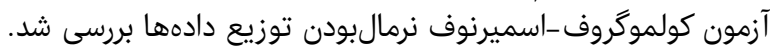

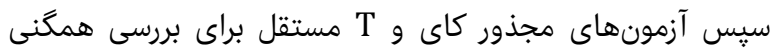

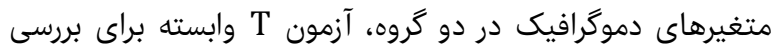

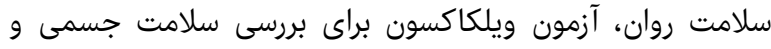

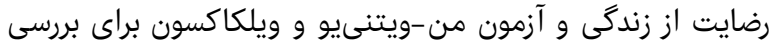

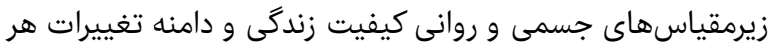
دو گروه مورد استفاده قرار گرفت.

\section{يافتهها}

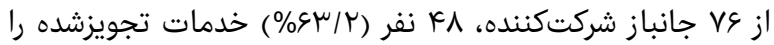

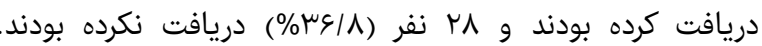

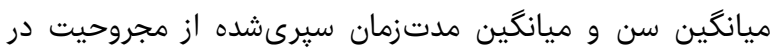

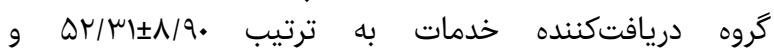
$\Delta Y / \Delta . \pm V / r \Delta$ Q و و

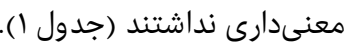

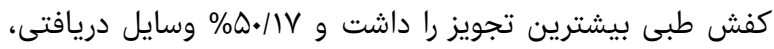

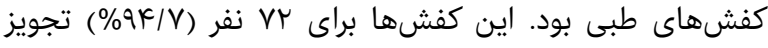




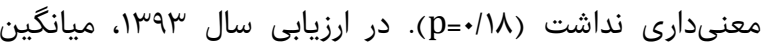

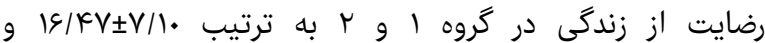

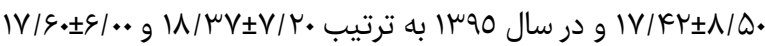

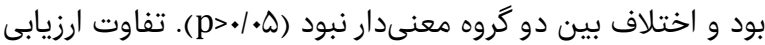

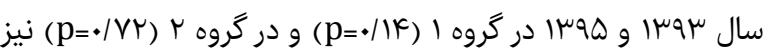

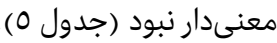
\% ج ج/9

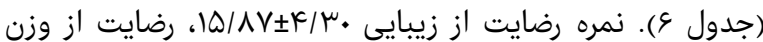
.

جدول 0) توزيع فراوانى مطلق و نسبى (اعداد داخل يرانتز درصد هستند) رضايت

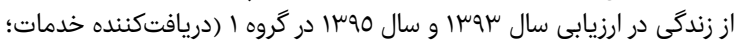

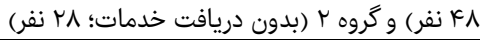

\begin{tabular}{|c|c|c|}
\hline سال هوس" & سال سqسו & ميزان رضايت \\
\hline & & كاملاً ناراضى \\
\hline $\mathcal{F}(\Lambda / \mu)$ & $\wedge(\mid \& / \vee)$ & كروها \\
\hline$r(V / I)$ & $V(r \Delta / \cdot)$ & r \\
\hline & & 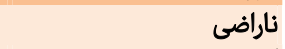 \\
\hline $\mathbb{I}(r V /)$ & $\mathbb{I r}(r \Delta / \cdot)$ & كروها \\
\hline$\Delta(I V / 9)$ & $\mu(1 . / V)$ & r \\
\hline & & كمى راضى \\
\hline $\mathbb{I r}(r \Delta / \cdot)$ & $11(r Y / 9)$ & كروها \\
\hline$\|\left({ }^{\mu q / \mu}\right)$ & $G(Y I / K)$ & كروه r \\
\hline & & عدم رضايت و عدم نارضايتى \\
\hline $1(r / 1)$ & $r(\kappa / r)$ & كروها \\
\hline $1(\mu / \varepsilon)$ & $\cdot(\cdot / \cdot)$ & كروه r \\
\hline & & كمى راضى \\
\hline $1 \cdot(r \cdot / \Lambda)$ & $1 \cdot(r \cdot / \Lambda)$ & كروها \\
\hline$G(Y / K)$ & $G(Y I / F)$ & كروه r \\
\hline$G(\mid r / \Delta)$ & $\mathcal{K}(\Lambda / \mu)$ & رَروها \\
\hline$\mu(1 . / v)$ & $\Delta(I V / Q)$ & كروه r \\
\hline & & بسيار راضى \\
\hline$r(r / r)$ & $1(Y / 1)$ & كروها \\
\hline$\cdot(\cdot / \bullet)$ & $1(\mu / \varphi)$ & r \\
\hline
\end{tabular}

جدول \&) توزيع فراوانى مطلق و نسبى (اعداد داخل يرانتز درصد هستند) ميزان

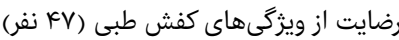

\begin{tabular}{|c|c|c|c|c|c|}
\hline راضىام & راضىام & نظر خاصى & نيستم & اصلاً راضى & كفش طبى طهاى \\
\hline $10(\mu / / q)$ & $\mu^{\mu}(F \wedge / q)$ & $\mu(\varepsilon / \mathcal{F})$ & $\mu(\varepsilon / \mathcal{k})$ & $\mu(\varepsilon / \kappa)$ & رنظ \\
\hline $10(\mu / / q)$ & $K F(\Delta I / I)$ & $\mu(\varepsilon / \mathcal{F})$ & $r(\kappa / r)$ & $\mu(\varepsilon / \mathcal{F})$ & 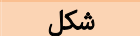 \\
\hline $10(\mu \mid / q)$ & $r \Delta(\Delta \mu / r)$ & $r(\kappa / \mu)$ & $r(r / \mu)$ & $\mu(\varepsilon / \mu)$ & صدا \\
\hline $10(\mu / / q)$ & KF (QI/I) & $r(r / r)$ & $\mu(\xi / \xi)$ & $\mu(\varepsilon / \xi)$ & ظاهر \\
\hline $10(\mu \mid / q)$ & $r \cdot(K r / q)$ & $I(T / 1)$ & $8(I K / \Lambda)$ & $\Delta(1.19)$ & وزن ع وزن \\
\hline $\mathbb{I F}(r q / \Lambda)$ & $r \cdot(K r / Q)$ & $r(K / r)$ & S (IK/A) & $\Delta(1.19)$ & كارآيى \\
\hline IV (WG/Y) & $r r(K G \mid \Lambda)$ & $\mu(\xi / \mathcal{k})$ & $\mu(\varepsilon / 4)$ & $r(r / r)$ & اطمينانبودن \\
\hline IV (TG/Y) & $r r(K \& \mid \Lambda)$ & $I(r / I)$ & $F(\Lambda / \Delta)$ & $\mu(\varepsilon / \mathcal{F})$ & متناسببودن \\
\hline IV (WG/T) & $M I(K K / V)$ & $1(r / I)$ & $\Delta(1.18)$ & $\mu(\varepsilon / \mathcal{R})$ & راحتبودن \\
\hline$\| F(r q / \Lambda)$ & $r r(K \varepsilon \mid \Lambda)$ & $r(r / r)$ & $\varepsilon(I r / \Lambda)$ & $\mu(\varepsilon / \mathcal{R})$ & رضايت كلى \\
\hline
\end{tabular}

بحث

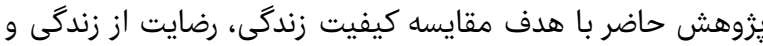

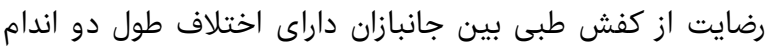

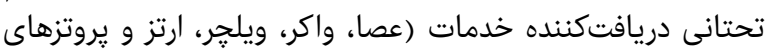

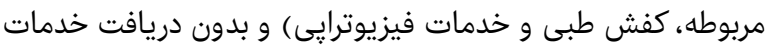

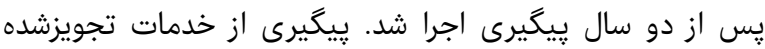

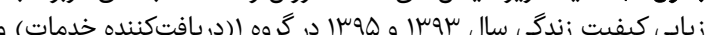

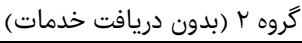

\begin{tabular}{|c|c|c|c|}
\hline سطح معنىدارى & $11 \% 90$ & سال سوسו & متغير \\
\hline \multicolumn{4}{|c|}{ زيرمقياس سلامت روان (MCS) } \\
\hline...$r$ & $r v / q r_{ \pm}+r \varepsilon / . \cdot$ & $k q / / k \pm r \mu / .$. & كروها \\
\hline.$\%$ & $\mu \kappa / q 1 \pm r k / \mu$. & $\Delta \Lambda / \kappa \mu \pm r \mu / q$. & كروه \\
\hline \multicolumn{4}{|c|}{ زيرمقياس سلامت جسمى (PCS) } \\
\hline $.1 . .1$ & $\mu \kappa / \varepsilon \mu_{ \pm} \mu \kappa / 1$. & $r \cdot\left|\lambda \mu_{ \pm} r \cdot\right| \Lambda$. & كروها \\
\hline.$|k|$ & $r \Delta / \wedge q \pm r \mu / Q$. & $r \mu / / \mu \pm r \cdot / q$. & كروه r r \\
\hline \multicolumn{4}{|c|}{ نمره كلى كيفيت زندگى } \\
\hline .18 & 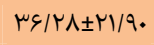 & $\mu F / Q \Lambda \pm 1 Q / \Delta$. & كروها \\
\hline.$/ . r$ & $\mu \cdot / \mu \cdot \pm r \cdot / \gamma$. & $k \cdot / V \wedge \pm 19 / V$. & كروه r \\
\hline
\end{tabular}

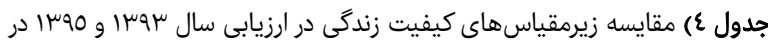

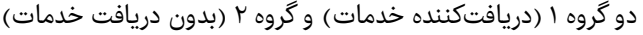

\begin{tabular}{|c|c|c|c|c|}
\hline معنىدارى سطح & تغييرات & سال هوسו & سال سوسו & متغيرها \\
\hline \multirow{4}{*}{$\begin{array}{l}. / Y K \\
. / 1\end{array}$} & \multicolumn{4}{|c|}{ سلامت عمومى (GH) } \\
\hline & $\mu / N \pm r r / r$. & $r \mu / K \mu \pm I V / K$. & $r \cdot / \mu 1 \pm 19 / \cdot \cdot$ & كروها \\
\hline & $-\Delta / \mu c \pm I V / 1$. & $1 \pi / \mu q \pm 1 \Delta / q$. & $|\wedge / \vee \Delta \pm| \xi / \mid$ & كروه r \\
\hline & & $.1 \cdot 1$ & ./А૬ & معنىدارى سطح \\
\hline \multirow{4}{*}{$.1 . .1$} & \multirow{4}{*}{ 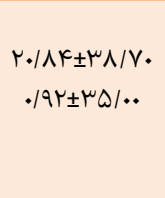 } & \multicolumn{3}{|c|}{ عملكرد فيزيكى (PF) } \\
\hline & & $\Delta \cdot / \Delta r \pm \mu r / \cdot \cdot$ & $r q / \varepsilon \wedge \pm r q / q$. & كروها \\
\hline & & $\mu V / Q \cdot \pm \mu \omega / \varphi$. & $\mu \varepsilon / \mu_{ \pm} \mu^{\mu} / r$. & 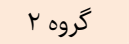 \\
\hline & & .11 & 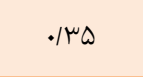 & معنىدارى سطح \\
\hline \multirow{4}{*}{$\begin{array}{l}\cdot / 14 \\
1 / \cdot \cdot\end{array}$} & \multirow{4}{*}{$• / . \pm<q / \cdot}$. & \multicolumn{3}{|c|}{ محدوديت در نقش فيزيكى (RP) } \\
\hline & & rN/ITEKR/G. & $\mid V / V \pm \Psi c / K$. & گروها \\
\hline & & $19 / \varepsilon \psi_{ \pm} \mu q / r$. & $19 / \varepsilon \mu_{ \pm} \mu q / r$. & كروه r \\
\hline & & . Irv &.$/ 9$ & معنىدارى سطح \\
\hline
\end{tabular}

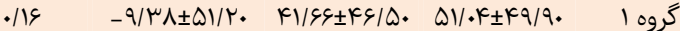

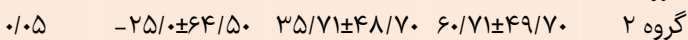

$\cdot / \Delta r$

معنى سطحارى

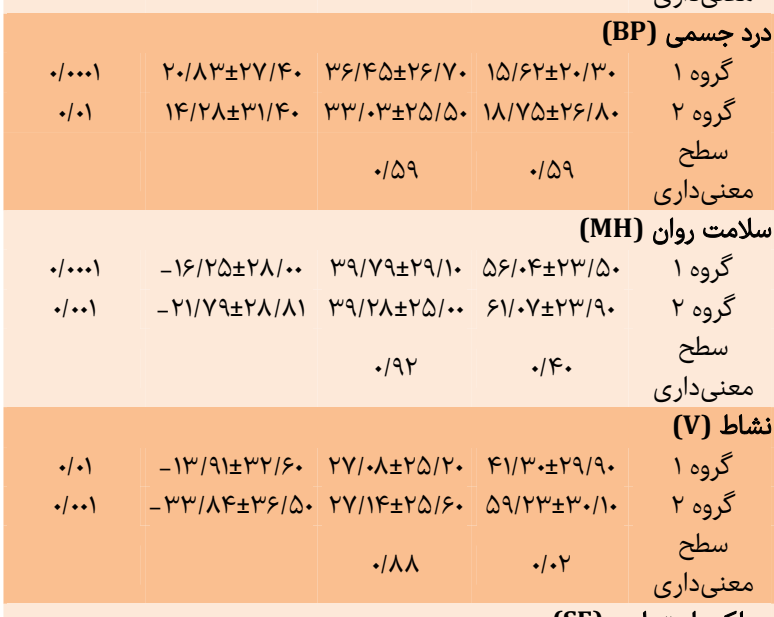

عملكرد اجتماعى (SF)

•

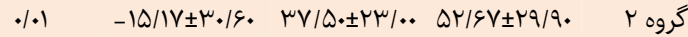

سطح

معنىدارى

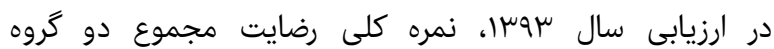

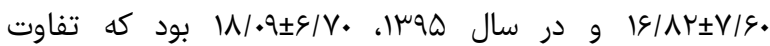


مقايسه كيفيت زندكى، رضايت از زندكى و رضايت از كفش طبى در بين جانبازان داراى اختلاف طول دو اندام تحتانى دريافتكننده ... اسا

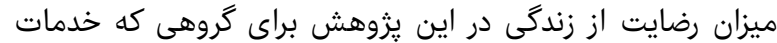

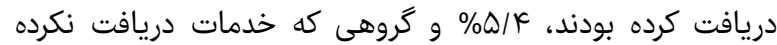

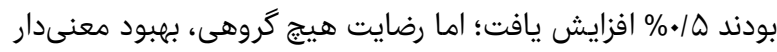

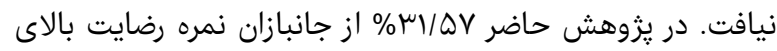

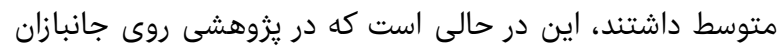

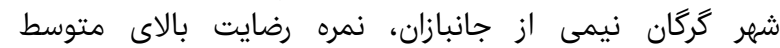

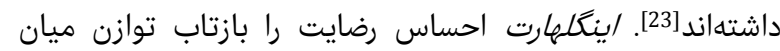

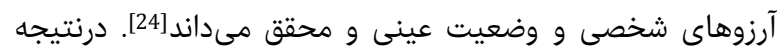

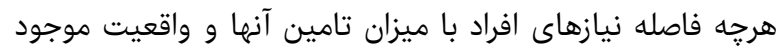

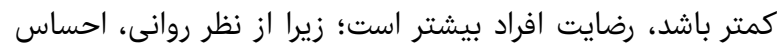

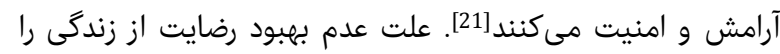

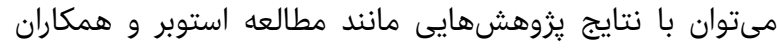

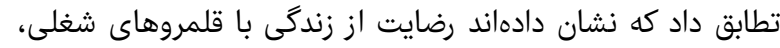

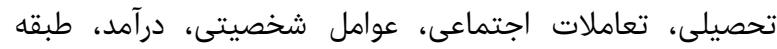

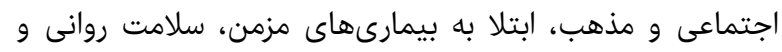

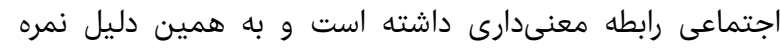

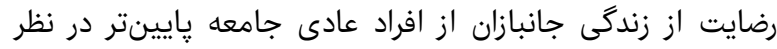

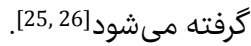

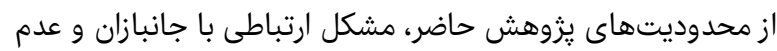

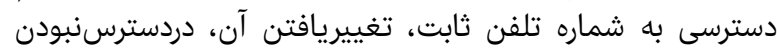

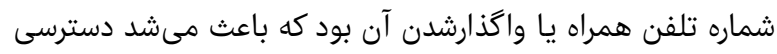

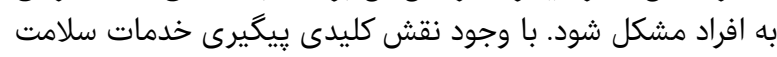

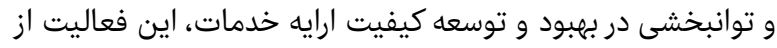

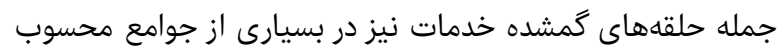

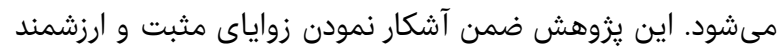

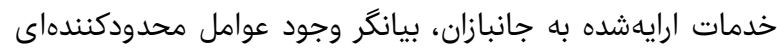

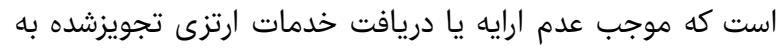

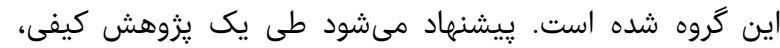
عوامل فوق بررسى شود.

\section{نتيجه}

اكثر جانبازان استفادهنننده از كفشهاي جان طبى رضايت بالايى دارند.

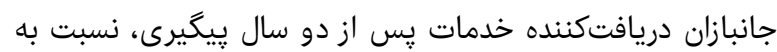

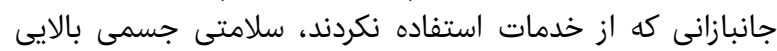

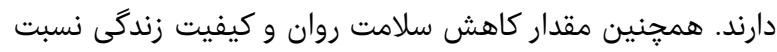

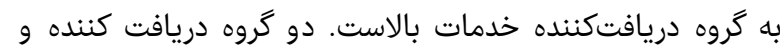

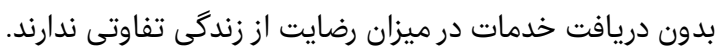

تشكر و قدردانى: از تمامى دوستان و عزيزانى كه ما را در اجراى اين

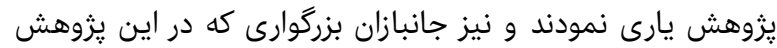

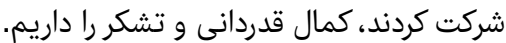

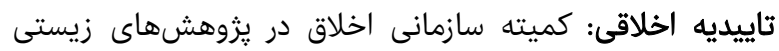

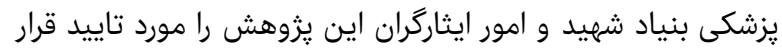

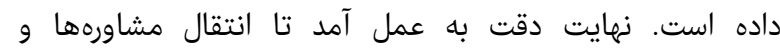

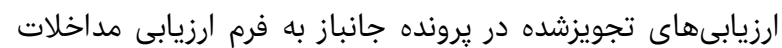

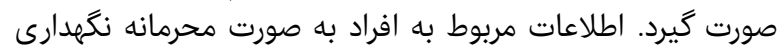

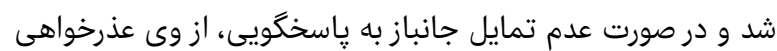
و از يزوهش خارج شدند.

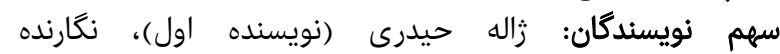

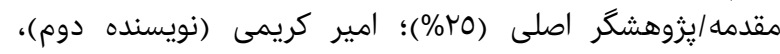

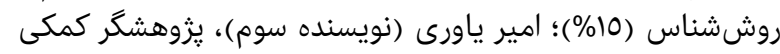

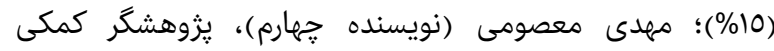

براى جانبازان با اختلال كوتاهى ڤا نشان داد كه

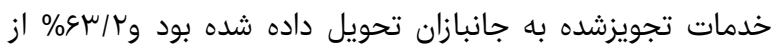

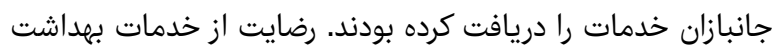

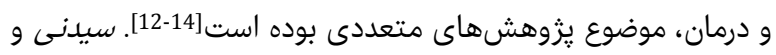

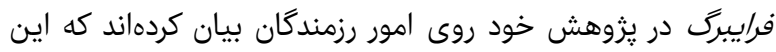

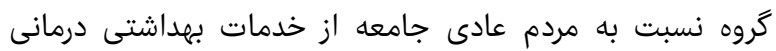

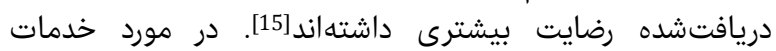

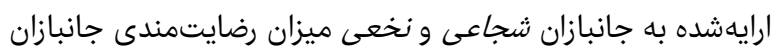

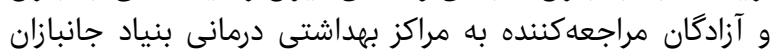

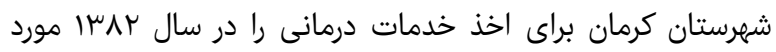

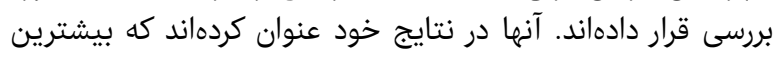

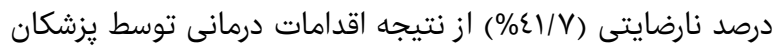

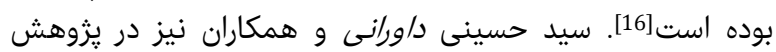

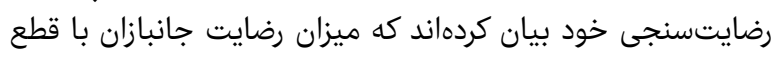

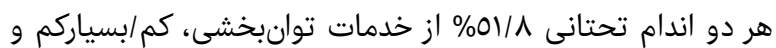

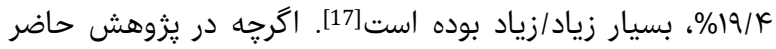

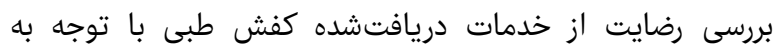

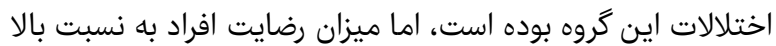

\section{بود.}

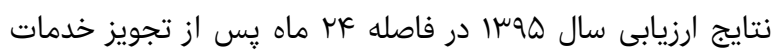

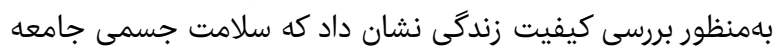

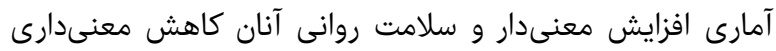

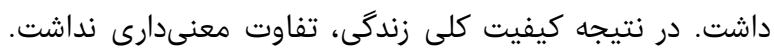

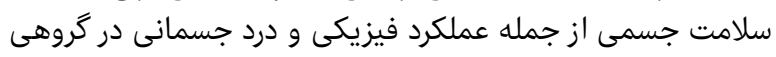

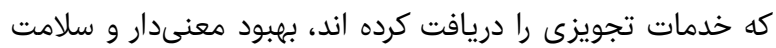

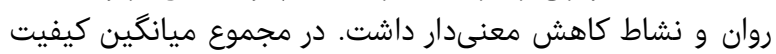

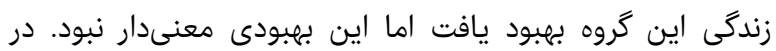

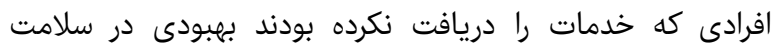

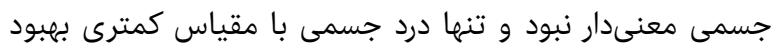

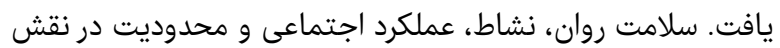

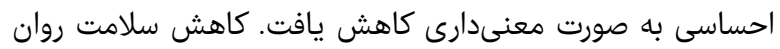

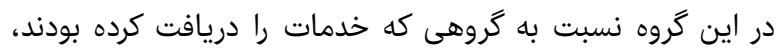

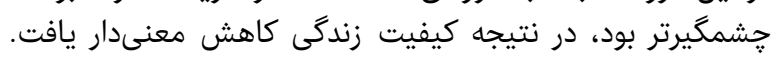

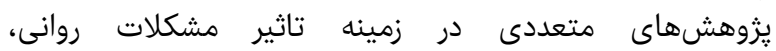

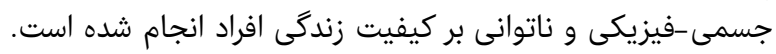

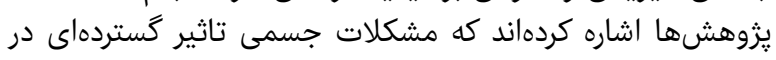

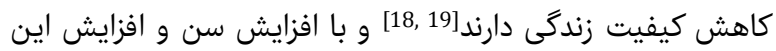

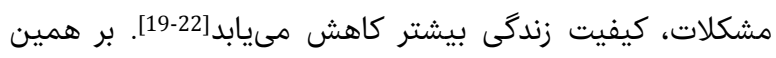

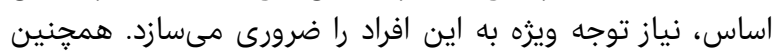

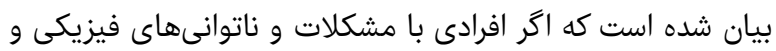

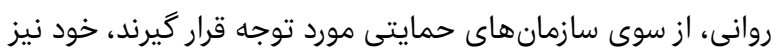

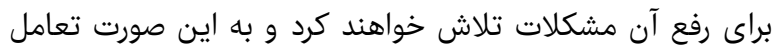

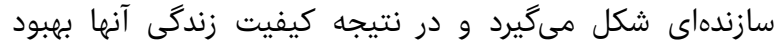

خواهد يافت [8].

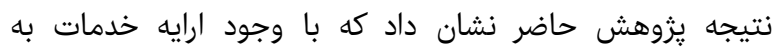

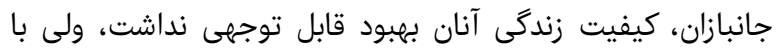

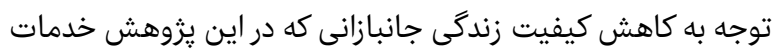

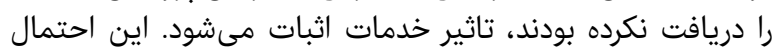

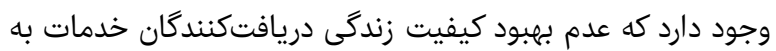

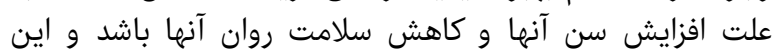

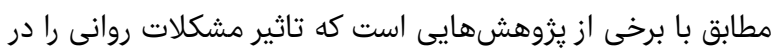

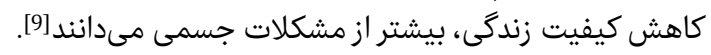


longitudinal study among patients served by the veterans health administration. Am J Med Qual. 2001;16(5):166-73.

15- Sydney J, Freedberg Jr. VA medical system earns high customer satisfaction ratings [Online News]. Government Executive; 2006.

16- Shojaei Baghini H, Nakhaee N. Satisfaction of janbazan and azadegan referring health center of janbazan foundation of Kerman city to rcceive medical services in the year 2003-2004. J Rafsanjan Univ Med Sci. 2005;4(1):41-48.

17- Seyed Hoseini Davarani SH, Mousavi B, KarbalaeiEsmaeili S, Soroush MR, Masoumi M. Service satisfaction among war related bilateral lower limb amputation. Iran J War Public Health. 2010;3(1):49-54. [Persian]

18- Kazis LE, Miller DR, Clark J, Skinner K, Lee A, Rogers W, et al. Health-related quality of life in patients served by the department of veterans affairs: Results from the veterans health study. Arch Intern Med. 1998;158(6):626-32.

19- Amini R, Haghani H, Masoumi M, Nakhaee M. Quality of life in blind war survivors. Iran J War Public Health. 2008;1(2):24-35.

20- Prieto-Flores ME, Fernández-Mayoralas G, RojoPérez F, Lardiés-Bosque $R$, Rodríguez-Rodríguez $V$, Ahmed-Mohamed K, et al. Sociodemographic and health factors explaining emotional wellbeing as a quality of life domain of older people in Madrid, Spain: 2005. Rev Esp Salud Publica. 2008;82(3):301-13. [Spanish]

21- Ahmadi Kh, Nejati V. Evaluation of quality of life in psychiatric veterans of Isfehan. Iran J War Public Health. 2010;2(4):13-7.

22- Jafari F, Guitynavard F, Soroush MR, Mousavi B. Quality of life in chemical war victims with sever pulmonary damage. Iran J War Public Health. 2012;4(1):46-52.

23- Tavalaei S, Habibi M, Asaari S, Ghaneei M, Naderi Z, Khateri S, et al. Quality of life in chemical veterans 15 years after exposure to mustard gas. J Behav Sci. 2007;1(1):17-25.

24- Inglehart R. Modernization and postmodernization: Cultural, economic, and political change in 43 societies. New Jersey: Princeton University Press; 1997.

25- Stoeber J, Stoeber FS. Domains of perfectionism: Prevalence and relationships with perfectionism, gender, age, and satisfaction with life. Person Individ Differ. 2009;46(4):530-5.

26- Dubey A, Agarwal A. Coping strategies and life satisfaction: Chronically ill patients' perspectives. J Indian Acad Appl Psychol. 2007;33(2):161-8. (10\%)؛ زيبا والى (نويسنده ينجم)، يزوهشكر كمكى (10\%)؛ الهار (10)

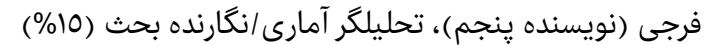

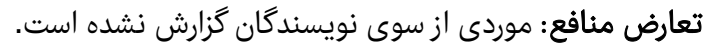

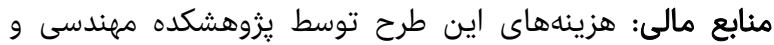
علوم يزشكى جانبازان تامين شده است.

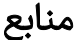

1- Johari A, Maheshwari R, Maheshwari S. Passport for the orthopedic boards and FRCS examination. Paris: Springer; 2015. p. 463-7.

2- Raczkowski JW, Daniszewska B, Zolynski K. Functional scoliosis caused by leg length discrepancy. Arch Med Sci. 2010;6(3):393-8.

3- McCarthy JJ, MacEwen GD. Management of leg length inequality. J South Orthop Assoc. 2001;10(2):73-85.

4- Stanitski DF. Limb-Length Inequality: Assessment and treatment options. J Am Acad Orthop Surg. 1999;7(3):143-53.

5- Goldberg B, Hsu JD. Atlas of orthoses and assistive devices. Maryland Heights: Mosby; 1997.

6- Hagberg K, Brånemark R, Hägg O. Questionnaire for persons with a transfemoral amputation (Q-TFA): Initial validity and reliability of a new outcome measure. J Rehabil Res Dev. 2004;41(5):695-706.

7- Nouraei MH, Javdan M, Nouraei F, Mohebbi Dehnavi A, Safdari F. study of stump problems of lower limb amputation in war casualties. Iran J Orthop Surg. 2014;12(2):47-51. [Persian]

8- Maslow AH. A theory of metamotivation: The biological rooting of the value-life. J Humanist Psychol. 1967;7(2):93-127.

9- Chen H, Cohen P, Kasen S, Johnson JG, Berenson K, Gordon K. Impact of adolescent mental disorders and physical illnesses on quality of life 17 years later. Arch Pediatr Adolesc Med. 2006;160(1):93-9.

10- Montazeri A, Goshtasbi A, Vahdaninia M. The short form health survey (SF-36): Translation and validation study of the Iranian version. 2006;5(1):49-56. [Persian] 11- Bayani AA, Kouchaki AM, Goudarzi H. The reliability and validity of the satisfaction with life scale. J Iran psychol. 2007;3(11):259-65.

12- Chisick MC. Satisfaction with military dental care by active duty soldiers. Mil Med. 1994;159(7):501-4.

13- Dunn WJ, Langsten RE. Dental satisfaction survey at an expeditionary dental clinic in support of operations enduring freedom and Iraqi freedom. Mil Med. 2004;169(10):773-6.

14- Ren XS, Kazis L, Lee A, Rogers W, Pendergrass S. Health status and satisfaction with health care: A 